\title{
城市代谢效率研究的热点识别与综合效率实证研究
}

\author{
武昊䑣 ${ }^{1,2}$, 洗超凡 ${ }^{1, *}$, 刘晶茹 ${ }^{1,2}$ \\ 1 中国科学院生态环境研究中心城市与区域生态国家重点实验室, 北京 100085 \\ 2 中国科学院大学, 北京 100049
}

摘要:城市代谢是城市可持续发展研究的重要部分,其有序运行为城市发展与居民生活提供重要保障,代谢效率的提高有助于 缓解资源利用与环境保护间的矛盾。通过分析国内外近 30 年的城市代谢效率的研究进展,整合代谢效率研究热点选取综合效 率评估所需指标, 并以深圳市为案例研究, 结合数据包络分析 (DEA) 模型对城市物质代谢综合效率进行评估。结果表明: ( 1) 近 30 年城市代谢研究处于蓬勃发展阶段, 中国在相关研究领域中的影响力凸显, 而城市代谢效率研究主要关注能源、水资源、 碳氮元素及耐用物质 (主要为建筑材料)等要素及其环境效应; (2) 基于上述要素对深圳城市代谢的综合效率进行评估,其综合 效率在 2005-2017 年间呈波动状态, 多数年份城市代谢过程取得了高效运行 (效率值大于 1),但仍存低效年份,包括 2006 （0.96）、2007（0.76）、2011(0.85) 和 2013(0.97) 年,研究期间综合效率提高不明显。在资源投人与效益产出方面,深圳市在水资 源利用效率方面需进一步提高; 在当前资源消耗水平下经济效益仍有提高潜力; 在减少碳氮元素的环境排放及建筑垃圾产生的 效率方面有一定程度的提高。(3) 通过城市代谢效率研究的热点识别, 可明晰目前城市代谢综合效率评估所需涵盖的要素, 由 此建立评估体系可用于综合效率评估的实践,通过案例分析研究其反映城市资源利用效率动态的可行性, 以期为城市可持续发 展评估提供科学参考。

关键词: 城市代谢; 文献计量;数据包络分析;效率评估;深圳市

\section{Hotspots identification for the research on urban metabolism efficiency with a case study addressing integrated efficiency}

WU Haotong ${ }^{1,2}$, XIAN Chaofan ${ }^{1, *}$, LIU Jingru ${ }^{1,2}$

1 State Key Laboratory of Urban and Regional Ecology, Research Center for Eco-Environmental Sciences, Chinese Academy of Sciences, Beijing 100085, China

2 University of Chinese Academy of Sciences, Beijing 100049, China

\begin{abstract}
Urban metabolism is long-term regarded as a hot topic in the research field of urban sustainability, and it's wellrunning provides an important guarantee for urban development and residents' livelihood. The improvement in metabolic efficiency helps to alleviate the contradictions of urban resource utilization and environmental protection. This research firstly analyzed the worldwide progresses in the research of urban metabolic efficiency over past 30 years, and then summarized the research hotspots of metabolic efficiency accompanied with indicators selection for integrated efficiency evaluation. Take the populous Shenzhen city in China as case study, which is regarded as the hotspots for urban metabolism research, and the Data Envelopment Analysis ( DEA) model was adopted to evaluate the integrated efficiency of urban metabolism. The results showed that: (1) The booming in urban metabolism research were emerging in the past 30 years, which contributed most by
\end{abstract}

基金项目: 国家自然科学青年基金项目 (42101290) ; 国家自然科学基金面上项目(71874181); 深圳市陆域生态调查评估(SZCG2018161498)

收稿日期: 2021-05-31；接收日期: 2021-09-28

*通讯作者 Corresponding author.E-mail: cfxian@ rcees.ac.cn 
China, especially in 2011-2020, followed by America, England and Italy. Research on urban metabolic efficiency mainly focused on the urban metabolism of energy, water resources, carbon element, nitrogen element and durable substances (mainly building materials) and their environmental effects. (2) Based on the evaluation of the integrated efficiency concerning above factors, the integrated efficiency of Shenzhen's urban metabolism presented fluctuated. In most years, the urban metabolic processes have achieved efficient operations, which were reflected by efficiency value $>1$, but there still existed inefficient years, including 2006 ( efficiency value=0.96), 2007 (efficiency value=0.76), 2011 ( efficiency value $=0.85)$ and 2013 (efficiency value=0.97), and the integrated efficiency of urban metabolism in Shenzhen has not been significantly improved during the study period. In terms of the benefit outputs from the process of resource inputs, the utilization efficiency of water resource in Shenzhen still needs to be improved. Based on current level of resource consumption, it is potential for Shenzhen to further rise economic benefits. However, the efficiencies of reductions in carbon and nitrogen emissions, as well as construction wastes, have been improved to a certain extent during study period. (3) Hotspots identification in the research of urban metabolism efficiency can clear elements of urban metabolism for the integrated efficiency evaluation, which contributes to the practical of integrated efficiency evaluation with the established evaluation system. The case study showed that the feasibility of integrated efficiency evaluation of urban metabolism to reveal the dynamic in overall resources use efficiency in a city, aiming at providing scientific reference for the assessment of urban sustainability towards national sustainable development.

Key Words : urban metabolism; bibliometric; data envelopment analysis; efficiency evaluation; Shenzhen

随着城市化的快速发展, 城市代谢相关研究日趋丰富 ${ }^{[1]}$ 。城市代谢的概念最早于 1965 年由 Wolman 提 出,他将城市的运行比作生物体代谢,认为城市代谢与生物代谢相似,都是从外界环境摄取物质而后向环境输 出废弃物的过程 ${ }^{[2]}$ 。1988 年 Robert Ayres 确立了物质代谢的最初研究范畴 ${ }^{[-4]}$ 。2001 年 Harberl 结合物质流 与能量流分析方法用于城市物质代谢研究 ${ }^{[5-6]}$, 此后城市代谢的研究方法逐渐统一。相关研究除了关注城市 代谢与外界交互的循环过程, 也开始通过模型建立来分析代谢系统的内部循环特征, 将过程和节点转换为数 学语言以明确城市代谢物质、能量流的特性并进行优化 ${ }^{[7-9]}$ 。经过多年发展, 城市代谢理论及其分析框架已 被广泛应用于分析城市人口、资源与环境的相互关系, 识别城市生态系统的内部过程 ${ }^{[10]}$, 以寻求其中的冲突 及潜在问题最优解决方法。

城市代谢效率是城市代谢研究的重点内容, 其可为城市可持续发展提供科学参考 ${ }^{[11]}$ 。城市代谢效率的 概念来自于生态效率, 经济合作与发展组织将其定义为: 生态资源用于满足人类需求的效率 ${ }^{[12]}$, 广义来看是 系统产出与投人之比 ${ }^{[13]}$ 。由于城市代谢过程涉及社会、经济与自然子系统之间复杂的物质能量流动, 因此大 部分研究都采取指标体系法和模型分析法来分析城市代谢效率 ${ }^{[13]}$ 。在研究方法方面, 张妍等 ${ }^{[11]}$ 综合环境效 率与资源效率构建了城市生态效率的度量模型; 同时, 也有学者 ${ }^{[7]}$ 结合能值分析法, 对城市物质代谢通量、存 量及效率进行分析。数据包络分析 (Data Envelopment Analysis, DEA) 是一种能满足多投人多产出指标的模 型, 其将评价对象作为一个生产决策单元 (Decision Making Units, DMU), 能够整合系统的投人和产出指标进 行效率运算, 常被用于城市代谢效率评估。如相关学者运用 DEA 模型针对不同形态/规模城市的代谢效率进 行分析, 旨在从空间形态上优化城市代谢效率 ${ }^{[14]}$; 有学者运用能值分析结合 DEA 模型对我国典型城市的代 谢效率特征及发展机制进行分析, 旨在实现对城市资源代谢的调控 ${ }^{[15]}$; 也有学者通过 DEA 模型评价不同城 市的元素代谢过程及其污染减排效率 ${ }^{[16-17]}$ 。目前相关研究采用的投人指标多以自然、社会与经济资源等指 标为主, 而产出指标常选取社会经济效益等期望产出指标或以废水、废气和废渣为代表的非期望产出指标, 其 主要是从资源投人是否能产出更大的社会经济效益或更少的污染排放角度来阐释城市代谢效率的高低, 在指 标类型的选择与数量上并没有统一的标准。同时,大部分研究多关注不同城市代谢效率间的横向比较,较少 纵向分析城市不同要素代谢效率的动态变化及其对城市代谢整体状况的影响, 难以反映当前城市代谢模式下 
的综合效率。为了解城市代谢综合效率评估所应涵盖的要素,有必要通过分析城市代谢效率研究进展与发展 趋势、识别研究热点及其涉及的要素,从中选出能反映城市代谢综合效率的代表指标。

CiteSpace 是一款可用于科学文献信息挖掘与可视化的应用程序 ${ }^{[18]}$, 已用于多个领域的文献计量分 析 ${ }^{[19-21]}$ 。本文拟先通过 CiteSpace 对城市代谢的研究进展进行梳理, 识别城市代谢效率研究的热点,结合现有 研究热点所涵盖的代谢要素, 建立可反映城市代谢综合效率的指标体系, 以城市代谢研究热点地区深圳市为 案例研究,评估其城市代谢的综合效率,揭示其资源可持续利用程度, 以期为同类城市的综合效率评估提供方 法参考,从而能为城市代谢管理的实践提供科学支撑。

\section{1 研究方法}

\section{1 文献计量分析}

文献计量法于 1969 年首次被提出,是一种运用定量的统计分析工具对发表文献进行分析从而获得有价 值信息的量化分析方法 ${ }^{[22]}$ 。随着各学科研究快速发展, 越来越多的研究使用文献计量分析方法回顾领域内 研究热点与前沿, 并使之可视化 ${ }^{[22]}$, 使读者能够更直观地了解学科的研究内容, 更好地把握学科发展的整体 脉络。因此, 本研究先对城市代谢研究进行文献检索, 在检索结果的基础上加人代谢效率等关键词汇进行二 次检索。基于中国知网和 Web of Science 两个数据库,选取如物质流、能量流和元素流等城市代谢研究所涉 及的关键词汇作为主题进行检索, 时间跨度为 1990 年 1 月 1 日一-2020 年 12 月 30 日。经过篮选 ,最终在中国 知网中获得 723 篇文献,在 Web of Science 中获得 1574 篇文献,基于此再以 “代谢效率” 为主题进行二次检 索, 文献计量分析在 Citespace 5.7R5W 版本上实现。

\section{2 物质代谢综合效率分析}

\subsection{1 案例研究地区}

深圳作为第一批可持续发展创新示范市之一, 致力于通过实施资源高效利用为超大型城市可持续发展发 挥示范效应 ${ }^{[23]}$,但目前极度依赖进口资源来满足日益增加的人口与经济规模, 制约了城市的可持续发展。因 深圳市同时具有“资源、空间小市”与“人口、经济大市”等特点,往往被选为城市代谢研究的热门案例地区, 自 2000 年以来已有相关研究对深圳城市代谢状态进行分析 ${ }^{[11,24-25]}$, 研究对象涵盖多种要素代谢过程。早期涉 及城市代谢效率研究主要集中于能源与水等要素的消费及其污废排放, 后续相关研究逐渐关注于特殊要素 (如元素、信息、金属等) 代谢流的量化及其环境影响 ${ }^{[1,26-27]}$, 大量前人研究可为深圳城市代谢综合效率评估 的开展提供数据参考。

\subsection{2 数据包络分析 (DEA)}

数据包络分析 (DEA) 方法是基于线性规划模型来平行评价决策单元综合效率(具有相同类型的多投人 与多产出指标) 的一种非参数统计方法 ${ }^{[28]}$ 。现有研究大多采用传统基于不变规模报酬与可变规模报酬模型, 其主要能辨析出有效(效率值为 1 ) 与非有效单元 (效率值小于 1), 但无法对多个有效单元作进一步判别, 而 这对于表征时间序列的单元间比较无为重要。超效率数据包络分析模型的提出与改进 ${ }^{[29-30]}$,可通过基于修 正松他变量的 Super-SBM( slacks-based measure) 模型来处理效率评价中可能出现的多个决策单元同时有效 (效率值为 1) 而无法比较的情形, 进一步对 DEA 分析中大于 1 的有效单元继续进行评价和排序 ${ }^{[31]}$, 更深刻地 体现效率评价的本质 ${ }^{[30]}$ 。本文采用以上 Super-SBM 模型对城市代谢综合效率进行评估。该模型定义为假设 城市代谢系统有 $n$ 个决策单元, 每个决策单元代表特定年份, 都有 $m$ 个投人变量和 $t$ 个产出变量, 投人向量和 产出向量分别为 $x \in R^{m}, y \in R^{t}$, 定义矩阵 $X 、 Y$ 为:

$$
\begin{aligned}
X & =\left[x_{1}, \cdots, x_{n}\right] \in R^{m \times n}>0 \\
Y & =\left[y_{1}, \cdots, y_{n}\right] \in R^{t \times n}>0
\end{aligned}
$$

在 Super-SBM 模型中,排除了决策单元 $\left(x_{0}, y_{0}\right)$ 的有限生产可能性集 ${ }^{[31]}$ 为:

$$
p /\left(x_{0}, y_{0}\right)=\left\{(\bar{x}, \bar{y}) \mid \bar{x} \geqslant \sum_{j=1}^{n} \lambda_{j} x_{j}, \bar{y} \leqslant \sum_{j=1}^{n} \lambda_{j} y_{j}, \bar{y} \geqslant 0, \lambda \geqslant 0\right\}
$$


Super-SBM 规划模型可以表达为:

$$
\begin{aligned}
& \rho^{*}=\min \frac{1}{m} \sum_{i=1}^{m} \frac{\bar{x}_{i}}{x_{\mathrm{i} 0}} / \frac{1}{t} \sum_{\mathrm{r}=1}^{t} \frac{\bar{y}_{\mathrm{r}}}{y_{\mathrm{r} 0}} \\
& \text { s.t. } \bar{x} \geqslant \sum_{j=1, \neq 0}^{n} \lambda_{j} x_{j}, \bar{x} \geqslant x_{0} \\
& \bar{y} \geqslant \sum_{j=1, \neq 0}^{n} \lambda_{j} y_{j}, \bar{y} \leqslant y_{0} \\
& x_{0}=X \lambda+s^{-}, s^{-} \geqslant 0 \\
& y_{0}=Y \lambda-s^{+}, s^{+} \geqslant 0 \\
& \bar{y} \geqslant 0, \lambda \geqslant 0
\end{aligned}
$$

式中, $\rho$ * 是决策单元超效率值, $\lambda$ 为权重向量。下标 “ 0 ” 表示参与评价的对应决策单元 ${ }^{[31]}, x_{0}$ 和 $y_{0}$ 分别为待 评单元的投人与产出向量, $x_{i 0}$ 和 $y_{r 0}$ 为 $x_{0}$ 和 $y_{0}$ 的元素; $m 、 t$ 分别为投人、产出要素数量。城市代谢过程往往需 要资源投人来获得产品产出从而获得效益,这不可避免地导致了污染物排放等非期望输出, 在评估过程中可 采取非期望输出取倒数法处理非期望指标 ${ }^{[16-17]}$, 强调如何以更少资源消耗获得较大的期望产出与较小的非 期望产出。 $S^{-} 、 S^{+}$分别代表松弛投人量和松弛产出量, 其数量级影响着决策单元的最终效率, 松弛变量越少且 小表征决策单位综合效率越高。在此模型中, 当 $0 \leqslant \rho^{*}<1$, 则被评单元被视为无效, 在城市代谢综合效率方 面表现为低效, 存在进一步提高的空间, 当 $\rho^{*} \geqslant 1$ 时, 被评价单元有效, 其在综合效率方面表现为相对高效。

\section{2 结果}

2.1 城市物质代谢效率研究进展及热点

通过 Web of Science 以城市代谢相关的关键词进 行检索可知, 近 30 年来城市代谢研究一直稳步发展, 全 球英文文献数量逐年上升,截至 2020 年总量达到 1574 篇, 且仍具有增长可能(图 1)。城市代谢研究相关的英 文文献大多发表在生态环境科学与资源利用 $(60.17 \%)$ 、工程学 $(44.28 \%)$ 、科学技术 $(25.92 \%)$ 、燃料 能效 $(11.82 \%)$ 、水资源利用 $(7.75 \%)$ 以及结构与建筑 技术 $(6.67 \%)$ 等领域。可知国际刊物对于城市代谢研 究更关注基础理论, 聚焦于生态环境、土木工程、系统科 学等方面, 且常涉及多学科交叉, 形成不断丰富的研究 领域。
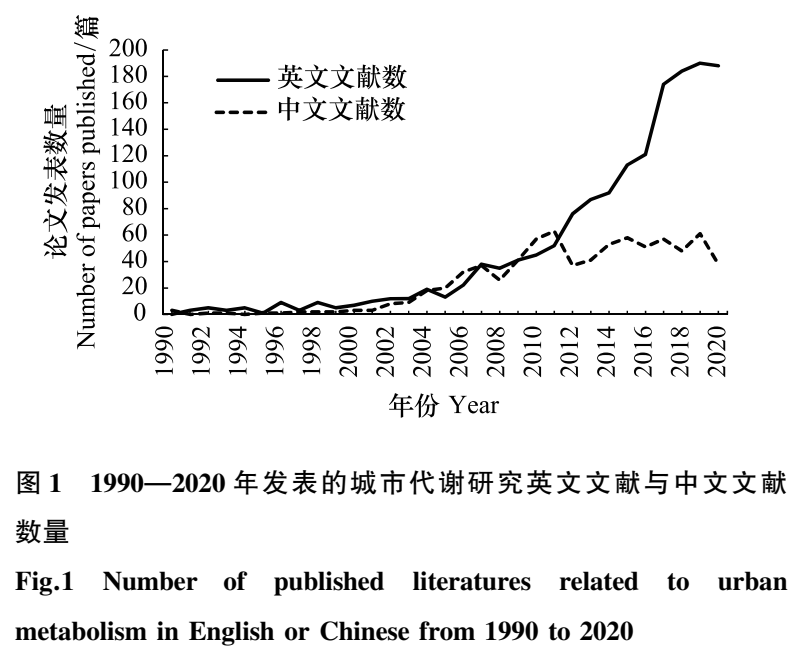

从英文文献发表数量来看, 中国学者发表的英文文 献以 435 篇的数量位列第一,近 30 年来其论文发表总数占比高达 $27.64 \%$ 。美国、英国、意大利位列第二至第 四名。值得一提的是,在 1990-2010 年的 20 年中,中国学者发表的英文论文数量仅有 45 篇,仅占该时段论 文总数 $15.15 \%$ 。而在 2011-2020 年, 中国的论文发表数大幅提升,这十年间本研究领域超过三分之一的论 文来自中国, 成为本领域文章发表最多的国家。这表明中国的城市代谢研究起步虽晚,但是发展速度迅猛。 中国的研究机构发文数量遥遥领先, 表明我国在城市代谢领域研究已有较强的国际影响力。

对由中国知网检索得到的 723 个结果分析可知,近 30 年城市代谢相关研究的中文文献发表经历了波动 增加趋势 (图 1), 主要集中在 2000-2011 年阶段,此后发文量保持相对稳定, 未出现上涨趋势, 与英文文献发 表量差距越来越大,2020 年仅为英文文献数量的五分之一。物质代谢相关的中文论文多关注环境科学与资 源利用 $(29.16 \%)$ 、宏观经济管理与可持续发展 $(21.31 \%)$ 、建筑科学与工程 $(14.42 \%)$ 等领域。综上所述,环境 科学与资源利用领域为全球城市代谢研究论文发表最多的领域, 说明城市环境与资源问题仍是城市代谢研究 
关注的重点。

在涉及城市代谢研究的文献里，“代谢效率”这一 关键词汇在中文文献中表述不多,其在部分文献多以 “代谢研究”、“排放强度”、“利用效率”等类似词汇体 现,而在英文文献中代谢效率研究大多包含“urban metabolism”、“efficiency”等关键词,表达较为一致,加 之涉及城市代谢效率分析的英文文献总量较多, 导致中 文与英文文献检索数量差别较大。因此,增添“代谢效 率”作为关键词, 对相关英文文献作进一步检索, 并对 篮选的文献进行聚类分析可知 (图 2,表 1), 当前城市 物质代谢效率的研究热点主要涉及城市资源消费效率 及其可持续发展,分析要素集中于能源、碳氮元素及耐 用物质 (主要为建筑材料) 等, 研究内容多关注物质能 量代谢、产业生态、污染排放等内容。

结合文献分析可知,城市代谢过程通常由水资源、 化石能源、营养元素以及若干耐用物质 (金属、木材与 石材等)在多个部门间的循环构成 ${ }^{[32]}$,这些要素在满足 了城市社会经济发展对资源消费的多元需求后,便以多 种形态进人环境,如能源消费产生的碳、氮元素多以气 体形态向环境排放 ${ }^{[8]}$, 而氮元素同时也通过食物与水

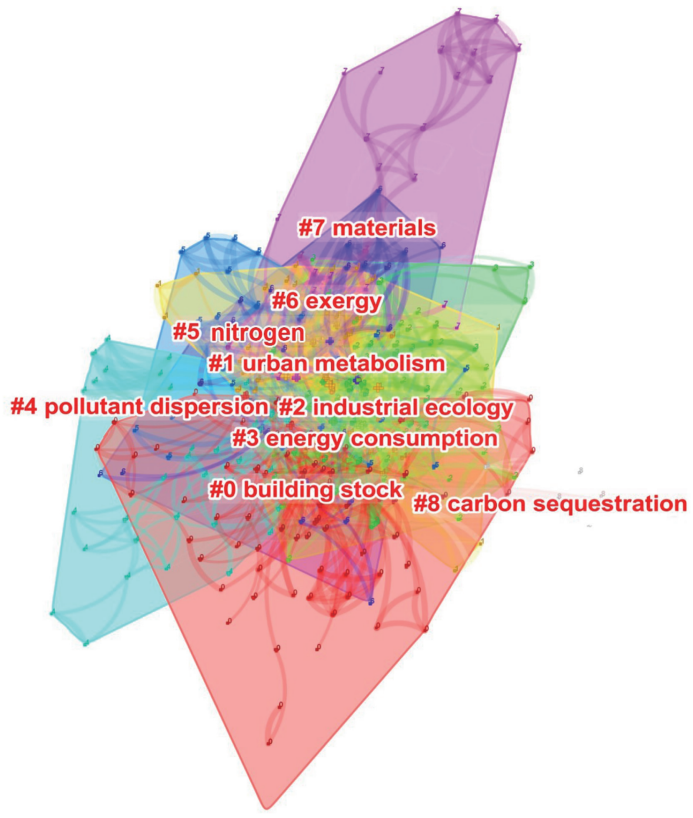

图 2 城市代谢效率相关文献聚类

Fig.2 Clustering of literatures related to urban metabolic efficiency 资源消费过程流失到水体 ${ }^{[33-34]}$, 耐用物质在城市环境中以固体形式存储和流转,成为物质存量或固废 ${ }^{[35]}$ 。而 现有效率分析大多针对其中一个或一类过程进行分析,少有研究包含上述多个投人产出过程进行综合效率分 析。本研究根据案例地区相关数据的可获得性,构建相关投人产出指标体系 (表 2) 来评估深圳市 2005一2017 年期间城市代谢的综合效率。指标数据来源于《2006-2018 年深圳统计年鉴》,《2005-2017 年深圳市水资 源公报》,深圳市政府部门官方网站以及近期发表的相关文献( 表 2)。

表 1 关键词聚类信息表

Table 1 Summaries of keyword clusters

\begin{tabular}{|c|c|c|c|c|c|c|}
\hline $\begin{array}{l}\text { 聚类号 } \\
\text { Clusters ID }\end{array}$ & $\begin{array}{l}\text { 大小 } \\
\text { Size }\end{array}$ & $\begin{array}{l}\text { 轮廓系数 } \\
\text { Silhouette }\end{array}$ & $\begin{array}{c}\text { 平均年份 } \\
\text { Mean( Year) }\end{array}$ & $\begin{array}{l}\text { 关键主题 (对数似然比 } / P \text { 值) } \\
\text { Top terms } \\
\text { (log-likelihood ratio/ } P \text {-level) }\end{array}$ & $\begin{array}{c}\text { 对数似然比 } \\
\text { Log-likelihood ratio }\end{array}$ & $P$ \\
\hline \multirow[t]{3}{*}{$\# 0$} & 77 & 0.751 & 2014 & building stock & 11.33 & 0.001 \\
\hline & & & & greenhouse gas emissions & 9.69 & 0.005 \\
\hline & & & & material flow & 8.37 & 0.005 \\
\hline \multirow[t]{4}{*}{$\# 1$} & 61 & 0.777 & 2012 & urban metabolism & 16.83 & 0.0001 \\
\hline & & & & ecological network analysis & 12.55 & 0.001 \\
\hline & & & & urban ecology & 12.01 & 0.001 \\
\hline & & & & water efficiency & 8.99 & 0.005 \\
\hline \multirow[t]{4}{*}{$\# 2$} & 42 & 0.735 & 2012 & industrial ecology & 13.25 & 0.001 \\
\hline & & & & energy use & 11.74 & 0.001 \\
\hline & & & & sustainable cities & 11.74 & 0.001 \\
\hline & & & & emission & 7.81 & 0.01 \\
\hline \multirow[t]{3}{*}{$\# 3$} & 40 & 0.782 & 2011 & energy consumption & 8.04 & 0.005 \\
\hline & & & & urbanization & 5.92 & 0.05 \\
\hline & & & & climate change & 5.92 & 0.05 \\
\hline
\end{tabular}




\begin{tabular}{|c|c|c|c|c|c|c|}
\hline $\begin{array}{c}\text { 聚类号 } \\
\text { Clusters ID }\end{array}$ & $\begin{array}{l}\text { 大小 } \\
\text { Size }\end{array}$ & $\begin{array}{l}\text { 轮廓系数 } \\
\text { Silhouette }\end{array}$ & $\begin{array}{c}\text { 平均年份 } \\
\text { Mean( Year) }\end{array}$ & $\begin{array}{l}\text { 关键主题(对数似然比 } / P \text { 值) } \\
\text { Top terms } \\
\text { (log-likelihood ratio/P-level) }\end{array}$ & $\begin{array}{c}\text { 对数似然比 } \\
\text { Log-likelihood ratio }\end{array}$ & $P$ \\
\hline \multirow[t]{2}{*}{$\# 4$} & 37 & 0.83 & 2012 & pollutant dispersion & 12.08 & 0.001 \\
\hline & & & & urban street canyon & 6.02 & 0.05 \\
\hline \multirow[t]{5}{*}{$\# 5$} & 34 & 0.718 & 2013 & nitrogen & 11.65 & 0.001 \\
\hline & & & & urban agglomeration & 6.33 & 0.05 \\
\hline & & & & food system & 5.81 & 0.05 \\
\hline & & & & waste management system & 5.81 & 0.05 \\
\hline & & & & environmental nitrogen load & 5.81 & 0.05 \\
\hline \multirow[t]{3}{*}{$\# 6$} & 30 & 0.906 & 2012 & exergy & 12.3 & 0.001 \\
\hline & & & & efficiency & 11.51 & 0.001 \\
\hline & & & & construction and demolition waste & 8.49 & 0.005 \\
\hline \multirow[t]{3}{*}{$\# 7$} & 28 & 0.84 & 2005 & materials & 7.16 & 0.01 \\
\hline & & & & urban material flow analysis & 5.41 & 0.05 \\
\hline & & & & environmental impact & 5.41 & 0.05 \\
\hline \multirow[t]{2}{*}{$\# 8$} & 9 & 0.99 & 2013 & carbon sequestration & 11.11 & 0.001 \\
\hline & & & & soil organic matter & 11.11 & 0.001 \\
\hline
\end{tabular}

注: 轮廓系数 ( silhouette) : 聚类相似性度量, 是簇的密集与分散程度的评价指标; 轮廓系数的值在 -1 和 1 之间, 该值越接近于 1 , 簇越紧凑, 聚类越好; 对数似然比 (log-likelihood ratio, LLR) : 聚类标签词提取算法之一, 在聚类里 LLR 越大的词越具有代表性

表 2 城市代谢综合效率的投入产出指标体系

Table 2 Input-output indicators for the integrated evaluation of urban metabolism

\begin{tabular}{|c|c|c|c|c|c|}
\hline $\begin{array}{l}\text { 指标类型 } \\
\text { Indicator }\end{array}$ & $\begin{array}{l}\text { 指标名称/单位 } \\
\text { Indicator name/Unit }\end{array}$ & $\begin{array}{l}\text { 最小值 } \\
\text { Minimum }\end{array}$ & $\begin{array}{c}\text { 最大值 } \\
\text { Maximum }\end{array}$ & $\begin{array}{c}\text { 标准差 } \\
\text { Standard } \\
\text { deviation }\end{array}$ & $\begin{array}{l}\text { 含义 } \\
\text { Definition }\end{array}$ \\
\hline 投人指标 & 用水量/亿 $\mathrm{t}$ & 16.77 & 20.16 & 1.02 & 城市年度用水总量 ${ }^{[36]}$ \\
\hline \multirow[t]{3}{*}{ Input indicators } & 工业标准煤消费量/亿 $\mathrm{t}$ & 0.10 & 0.17 & 0.03 & $\begin{array}{l}\text { 城市原煤、原油、天然气等能源年度工业消耗量总和, 单位换算 } \\
\text { 为标准煤 } 37]\end{array}$ \\
\hline & 耐用物质存量消费/亿 $\mathrm{t}$ & 2.03 & 3.62 & 0.51 & $\begin{array}{l}\text { 用于城市基础设施建设的金属、砾石与水泥等耐用物质年度存 } \\
\text { 量总和 }{ }^{227]}\end{array}$ \\
\hline & 食物消费量/万 $\mathrm{t}$ & 363.19 & 538.68 & 47.36 & $\begin{array}{l}\text { 城市居民各类食物年度消费总量, } 2005-2009 \text { 年采用 } 2009 \text { 年人 } \\
\text { 均食物消费量, } 2010-2017 \text { 年采用 } 2015 \text { 年人均食物消费量 }{ }^{[26]}\end{array}$ \\
\hline $\begin{array}{l}\text { 期望产出指标 } \\
\text { Desirable output indicators }\end{array}$ & GDP/亿元 & 4950.91 & 22490.06 & 5345.26 & 城市年度经济生产总值 ${ }^{[37]}$ \\
\hline $\begin{array}{l}\text { 非期望产出指标 } \\
\text { Undesirable output }\end{array}$ & 氮排放/万 $\mathrm{t}$ & 2.00 & 4.25 & 0.56 & $\begin{array}{l}\text { 城市年度活性氮排放 (含氮氧化物、氧化亚氮及氨气排放, 以及 } \\
\text { 水体点面源氮流失) }{ }^{[23]}\end{array}$ \\
\hline \multirow[t]{2}{*}{ indicators } & 碳排放/亿 $\mathrm{t}$ & 0.30 & 0.48 & 0.05 & 城市年度工业能源消耗碳排放 [38-39] \\
\hline & 建筑垃圾产生量/亿 t & 0.52 & 1.16 & 0.19 & 城市年度建筑与装修垃圾产量 ${ }^{[40]}$ \\
\hline
\end{tabular}

2.2 深圳城市代谢综合效率评估

结合 Super-SBM 模型与上述指标体系对深圳城市代谢综合效率分析可知 (表 3),2005-2017 年间综合效 率呈波动状态。相比 2005 年, 深圳城市代谢综合效率在 2017 年并没有得到明显提高。其中, 大部分年份表 现为 DEA 有效(效率值大于 1), 表明这些年份城市代谢过程取得了高效运行。2006、2007、2011 和 2013 年则 表现为 DEA 无效(效率值小于 1), 相对于其他有效年份, 这些无效年理论上可基于更少的资源消费量, 产出 更多的经济效益和减少更多的环境污染输出。通过投人产出松弛变量解读可揭示各年的资源投人几余量与 经济或减排产出不足量 (表 3), 在 2008-2012 年间,除了 2011 年,其余年份城市代谢综合效率均优且没有投 人攵余项。2011 年综合效率较低主要体现在当年资源利用效率不高, 如水、能源与建材等资源消费没有产出 理想的经济效益, 同时燃烧源温室气体与氮氧化物减排效率低。总体而言, 研究时期深圳城市代谢高效年份 
较多,然而,相比 2005 年,2017 年深圳城市代谢综合效率并没有得到明显提高。

表 3 城市代谢综合效率及其投入产出的几余与不足

Table 3 Integrated efficiencies of urban metabolism and the potential increases and decreases for their optimal inputs and outputs

\begin{tabular}{|c|c|c|c|c|c|c|c|c|c|}
\hline \multirow[b]{2}{*}{$\begin{array}{l}\text { 年份 } \\
\text { Year }\end{array}$} & \multirow[b]{2}{*}{$\begin{array}{c}\text { 效率值 } \\
\text { Efficiency } \\
\text { value }\end{array}$} & \multicolumn{4}{|c|}{ 投人圥余 Input surplus } & \multicolumn{4}{|c|}{ 产出不足 Output deficiency } \\
\hline & & $\begin{array}{c}\text { 用水量 } \\
\text { Water } \\
\text { consumption } \\
\left(1 \times 10^{4} \mathrm{t}\right)\end{array}$ & $\begin{array}{c}\text { 工业能源 } \\
\text { 消费量 } \\
\text { Industrial } \\
\text { energy } \\
\text { consumption } \\
\left(1 \times 10^{4} \mathrm{t}\right)\end{array}$ & $\begin{array}{l}\text { 食消费量 } \\
\text { Food } \\
\text { consumption } \\
\left(1 \times 10^{4} \mathrm{t}\right)\end{array}$ & $\begin{array}{c}\text { 耐用物质 } \\
\text { 存量消费 } \\
\text { Durable } \\
\text { material stock } \\
\text { consumption } \\
\left(1 \times 10^{4} \mathrm{t}\right)\end{array}$ & $\begin{array}{c}\text { GDP 增加量 } \\
\text { GDP growth } \\
\left(1 \times 10^{8} \mathrm{CNY}\right)\end{array}$ & $\begin{array}{l}\text { 碳排放 } \\
\text { 减少量 } \\
\text { Carbon } \\
\text { emission } \\
\text { reduction } \\
\left(1 \times 10^{4} \mathrm{t}\right)\end{array}$ & $\begin{array}{l}\text { 氮排放 } \\
\text { 减少量 } \\
\text { Nitrogen } \\
\text { emission } \\
\text { reduction } \\
\left(1 \times 10^{4} \mathrm{t}\right)\end{array}$ & $\begin{array}{c}\text { 建筑垃圾 } \\
\text { 减少量 } \\
\text { Construction } \\
\text { waste } \\
\text { reduction } \\
\left(1 \times 10^{4} \mathrm{t}\right)\end{array}$ \\
\hline 2005 & 1.08 & 0 & 0 & 0 & 654.27 & 0 & 468.07 & 0 & 569.07 \\
\hline 2006 & 0.96 & 1576.90 & 37.18 & 3.28 & 0 & 209.58 & 413.87 & 0 & 0 \\
\hline 2007 & 0.76 & 18323.63 & 313.78 & 37.71 & 0 & 800.55 & 969.58 & 1.24 & 0 \\
\hline 2008 & 1.02 & 0 & 0 & 0 & 0 & 0 & 0 & 0 & 442.43 \\
\hline 2009 & 1.01 & 0 & 0 & 0 & 0 & 0 & 174.64 & 0 & 0 \\
\hline 2010 & 1.04 & 0 & 0 & 0 & 0 & 0 & 0 & 0.29 & 0 \\
\hline 2011 & 0.85 & 4272.78 & 156.99 & 0 & 904.11 & 2417.49 & 148.77 & 0.70 & 0 \\
\hline 2012 & 1.04 & 0 & 0 & 0 & 0 & 0 & 0 & 0 & 1027.07 \\
\hline 2013 & 0.97 & 1145.55 & 0 & 1.60 & 72.32 & 1208.74 & 0 & 0.02 & 0 \\
\hline 2014 & 1.03 & 0 & 0 & 6.11 & 164.26 & 604.24 & 156.22 & 0 & 0 \\
\hline 2015 & 1.03 & 0 & 75.22 & 0 & 0 & 72.22 & 88.31 & 0 & 0 \\
\hline 2016 & 1.06 & 9013.29 & 34.57 & 0 & 0 & 1561.85 & 0 & 0.18 & 0 \\
\hline 2017 & 1.03 & 3801.02 & 0 & 0 & 0 & 2400.75 & 0 & 0 & 0 \\
\hline
\end{tabular}

相对于能源、食物与耐用物质消费,深圳市在水资源利用效率上仍存在提高空间,在研究时期水资源投人 圥余没有明显减少, 很大程度造成当年整体城市代谢低效; 而深圳在经济产出方面仍有潜力, 目前城市资源消 费并未获得最大经济效益,尤其是在 2011 年后,这一时期,深圳经济结构转型还在持续,在环境经济效益方面 仍未达到最优化阶段。对于污废排放,深圳市碳氮减排效率有显著提高,尤其 2007 年后深圳碳减排存在较少 产出不足年份, 且所需减排量也逐渐减少, 这归功于《深圳市国民经济和社会发展十一五规划纲要》强调的产 业结构优化与广推循环经济, 其促使减少化石能源消费及其相关污染排放成为环境管理工作重中之重 ${ }^{[40]}$, 从 而促进工业源温室气体与氮氧化物减排效率的提高。同时,深圳市保持相对少的建筑垃圾产生量,但在 2012 年建筑垃圾减少不足量达历年之最,这可能与当年高房价驱动下住宅扩建与旧城改造浪潮开始有关, 建筑拆 建与装修改造导致大量砖石、金属与木材等耐用物质报废为建筑垃圾 ${ }^{[41]}$ 。

\section{3 讨论}

目前深圳城市资源自给率很低,资源对外依赖性很大, 尤其是食物、能源、淡水、沙石木材等关键资源, 几 乎都需要区域之间的物质流动来支持深圳城市发展, 上述资源要素的可持续消费被认为是解决目前大型城市 人口膨胀、资源短缺和生态环境退化问题的关键所在 ${ }^{[42]}$ 。近年, 随着《粤港澳大湾区发展规划纲要》及《中共 中央国务院关于支持深圳建设中国特色社会主义先行示范区的意见》等文件相继出台,对深圳市可持续发展 的先行示范作进一步要求,其中对上述资源的可持续消费都分别作了针对性要求。因此,提高城市物质代谢 效率, 促进资源可持续消费, 对于深圳满足 “双区” 要求尤为重要。本文构建的指标体系能较好涵盖上述资源 要素, 可整体反映资源稀缺型城市的代谢状况。相较于早期针对深圳城市代谢效率的分析 ${ }^{[11]}$, 本文效率评估 方法不局限于单位 GDP 生产所需的水耗、能耗及其污废排放所构成的投人产出比值,而是整合多个关键要素 的代谢效率进行综合评估,评估结果能从整体视角为城市可持续发展提供科学参考。对于深圳市而言, 若以 单位 GDP 产出所需的资源消耗或污废排放来简单衡量城市代谢效率,往往易得出城市代谢效率逐步提高等 
结论, 毕竟深圳 GDP 增长率久位全国前列, 且其经济增长与资源消费已形成脱钩关系 ${ }^{[43]}$ 。相关前期研究也 通过 DEA 模型尝试结合多类投人产出项对深圳城市代谢效率及其环境效应进行一个整体刻画,但大都只关 注于废水、废气和固废等比较笼统的非期望输出指标,不能体现元素循环在城市食物、能源与水等必需资源消 费及代谢过程中的重要性 ${ }^{[42,44]}$ 。对于深圳市这类受土地资源约束的超大型城市, 其发展从 “增量扩张” 逐渐 转为“存量优化”阶段 ${ }^{[45]}$, 未来城市发展很大程度依赖于城市更新, 会加剧和扰乱城市耐用物质的代谢速率 及稳态, 城市代谢效率评估应需同时涵盖这一方面。

通过对城市代谢效率研究领域的热点识别, 从中选取关键指标形成综合效率评估指标体系, 既切合当前 城市代谢效率研究前沿, 又能相对满足城市可持续发展要求。联合国在《2030 年可持续发展议程》中提出了 17 项可持续发展目标 (Sustainable Development Goals, SDGs) ${ }^{[46]}$, 本评估体系可综合反映部分 SDGs, 如 SDG1 (消除饥饿), SDG6 (清洁饮水与卫生设施), SDG7 (能源普及与提高能源效率), SDG9 (基础设施与工业创 新),SDG11 (城市与社区可持续发展), SDG12 (可持续的消费与生产模式), 及 SDG13 (应对气候变化)。然 而,该体系所需指标易受数据不全或评价边界模糊影响, 导致应用范围受限。本文的结论提出仅是基于深圳 市 2005-2017 年的投人产出数据, 未来还需更长的时间序列数据以及不同地区的研究结果来验证方法的适 用性及其存在的误差。

城市代谢效率评估是保障城市生态系统有序运行的重要部分, 其能为城市决策部门优化资源配置, 提前 布局避免潜在生态环境问题提供科学参考,但从代谢效率评估阶段过渡到城市代谢优化阶段仍缺乏管理抓 手, 仍需相关方面的研究来促进城市代谢管理的有效 “落地”, 如城市代谢通量的常态化监测, 代谢过程的时 序模拟及空间化, 代谢效率评估的标准化及普适性等等。结合碳达峰、碳中和目标和探索 “无废城市” 建设将 成为未来深圳城市代谢效率研究的关注热点, 这为日后城市代谢综合效率评估提供了改善空间,使其更好服 务于未来城市代谢管理, 本研究对于综合效率评估方法的案例分析旨在为后续城市可持续发展评估迈出探索 的一步。

\section{4 结论}

本文通过文献计量分析, 梳理了国内外近 30 年的城市代谢效率的研究进展,整合了代谢效率研究热点所 涉及的要素, 以此建立评价指标体系通过案例研究对城市物质代谢综合效率进行评估。结果表明: (1) 环境 科学与资源利用领域为全球城市代谢研究论文发表最多的领域, 说明城市环境与资源问题仍是城市代谢研究 持续关注的重点。城市代谢效率研究热点关键要素包括能源、水资源、碳氮元素及耐用物质 (主要为建筑材 料) 等要素及其环境效应。(2) 通过案例分析, 深圳城市代谢综合效率在 2005-2017 年间呈波动状态, 多数 年份城市代谢过程取得了高效运行,但仍存低效年份,截止 2017 年, 综合效率并没有得到明显提高。在资源 投人与效益产出方面,深圳市在水资源利用效率方面仍需提高; 在当前的资源消耗水平下仍有较高的经济效 益提高潜力; 同时, 深圳市在碳氮元素环境排放及建筑垃圾减排减产效率也有了一定程度的提高。(3)通过 城市代谢效率研究的热点识别, 可明晰目前城市代谢综合效率评估所需涵盖的要素, 由此建立评估体系可用 于综合效率评估的实践运用,尽管仍需后续不断完善,但其能较全面反映城市资源利用效率的动态,可为日后 城市可持续发展评估提供科学参考。

\section{参考文献 ( References) :}

[1] 卢伊, 陈彬. 城市代谢研究评述: 内涵与方法. 生态学报, 2015, 35(8): 2438-2451.

[ 2 ] Wolman A. The metabolism of cities. Scientific American, 1965, 213: 179-190.

[ 3 ] Ayres R U. Industrial metabolism: theory and policy//Allenby B R, Richards D J, eds. The Greening of Industrial Ecosys Terns. Washington DC: National Academy Press, 1994.

[ 4 ] 王红娟. 张掖市城市代谢机理及优化调控研究 [D]. 兰州: 兰州大学, 2012.

[ 5 ] Haberl H. The energetic metabolism of societies part I: accounting concepts. Journal of Industrial Ecology, 2001, 5(1) : 11-33.

[ 6 ] Haberl H. The energetic metabolism of societies: part II: empirical examples. Journal of Industrial Ecology, 2001, 5(2) : 71-88. 
[ 7 ] Zhang Y, Yang Z F, Yu X Y. Ecological network and emergy analysis of urban metabolic systems: model development, and a case study of four Chinese cities. Ecological Modelling, 2009, 220(11): 1431-1442.

[ 8 ] Chen S Q, Chen B. Network environ perspective for urban metabolism and carbon emissions : a case study of vienna, austria. Environmental Science \& Technology, 2012, 46(8): 4498-4506.

[ 9 ] Zhang Y, Yang Z F, Yu X Y. Urban metabolism: a review of current knowledge and directions for future study. Environmental Science \& Technology, 2015, 49(19): 11247-11263.

[10] Wang X J, Zhang Y, Zhang J, Fu C L, Zhang X L. Progress in urban metabolism research and hotspot analysis based on CiteSpace analysis. Journal of Cleaner Production, 2021, 281: 125224.

[11] 张妍, 杨志峰. 城市物质代谢的生态效率——以深圳市为例. 生态学报, 2007, 27(8): 3124-3131.

[12] OECD. Eco-efficiency. Paris: Organisation for Economic Co-operation and Development, 1998.

[13] 尹科, 王如松, 周传斌, 梁菁. 国内外生态效率核算方法及其应用研究述评. 生态学报, 2012, 32(11): 3595-3605.

[14] 刘勇. 城市形态与城市物质代谢效率的相关性分析. 城市发展研究, 2010, 17(6): 27-31.

[15] 宋涛，蔡建明，倪攀，杨振山，温婷. 基于能值和 DEA 的中国城市新陈代谢效率分析. 资源科学，2013，35(11)：2166-2173.

[16] 冼超凡，王莉雁，逯非，郑华，欧阳志云. 基于 “氮补偿”机制及数据包络分析的活性氮减排评价一一以京津冀区域为例. 应用生态学报， $2017,28(8): 2545-2553$.

[17] 陆敏, 王增武. 基于 DEA 模型的中国碳排放管制效率研究. 生态经济, 2019, 35(6): 13-17.

[18] 陈悦, 陈超美, 刘则渊, 胡志刚, 王贤文. CiteSpace 知识图谱的方法论功能. 科学学研究, 2015, 33(2)：242-253.

[19］王耕, 周腾禹. 基于文献计量分析的区域生态安全研究热点与趋势. 生态学报, 2019, 39(18)：6950-6957.

[20］刘章生, 祝水武, 刘桂海. 国内生态资本文献计量研究. 生态学报, 2021, 41(4)：1680-1691.

[21] 王坤, 张丽君, 张超, 秦耀辰. 基于 CiteSpace 的生态城市发展研究动态分析. 生态学报, 2021, 41(5)：2097-2105.

[22] 郑文晖. 文献计量法与内容分析法的比较研究. 情报杂志, 2006, 25(5): 31-33.

[23] 冼超凡, 潘雪莲, 甄泉, 韩宝龙, 姜亚琼, 周伟奇, 欧阳志云. 城市生态系统污染氮足迹与灰水足迹综合评价. 环境科学学报, 2019, 39 (3) : 985-995.

[24] 宋涛, 蔡建明, 牛方曲, 杨振山, 倪攀. 基于 DEA 和主成分分析的中国城市代谢效率研究. 地理与地理信息科学, 2014 , 30(3) : 62-65.

[25] 陈雪婷, 宋涛, 蔡建明, 李玏, 邓羽. 基于 DEA 和 Malmquist 的中国城市代谢效率研究. 地理科学, 2015, 35(4)：419-426.

[26] 冼超凡, 刘晶茹, 潘雪莲, 欧阳志云. 典型移民城市食物氮足迹估算分析一一以深圳市为例. 生态学报, 2020, 40(20): 7441-7450.

[27] 李莹美, 陆轶峰, 刘晶茹, 洗超凡, 钱雨果. 深圳市城市化过程物质存量变化及驱动力研究. 环境科学与管理, 2020, 45(9): 165-169.

[28] 罗谷松, 李涛. 碳排放影响下的中国省域土地利用效率差异动态变化与影响因素. 生态学报, 2019, 39(13): 4751-4760.

[29] Tone K. A slacks-based measure of efficiency in data envelopment analysis. European Journal of Operational Research, 2001, 130(3): 498-509.

[30］刘勇, 李志祥, 李静. 环境效率评价方法的比较研究. 数学的实践与认识, 2010, 40(1): 84-92.

[31] 周泽昫, 胡建辉. 基于 Super-SBM 模型的低碳经济发展绩效评价研究. 资源科学, 2013, 35(12)：2457-2466.

[32] Kennedy C, Cuddihy J, Engel-Yan J. The changing metabolism of cities. Journal of Industrial Ecology, 2007, 11(2) : 43-59.

[33] Forkes J. Nitrogen balance for the urban food metabolism of Toronto, Canada. Resources, Conservation and Recycling, 2007,52 ( 1) : 74-94.

[34] Xian C F, Ouyang Z Y, Lu F, Xiao Y, Li Y M. Quantitative evaluation of reactive nitrogen emissions with urbanization: a case study in Beijing megacity, China. Environmental Science and Pollution Research, 2016, 23(17): 17689-17701.

[35] Miatto A, Schandl H, Forlin L, Ronzani F, Borin P, Giordano A, Tanikawa H. A spatial analysis of material stock accumulation and demolition waste potential of buildings: a case study of Padua. Resources, Conservation and Recycling, 2019, 142: $245-256$.

[36] 深圳市水务局. 深圳市水资源公报. 深圳: 深圳市水务局, 2005-2017.

[37］深圳市统计局，国家统计局深圳调查队. 深圳统计年鉴. 中国统计出版社, 2006-2018.

[38] Zhou Y, Shan Y L, Liu G S, Guan D B. Emissions and low-carbon development in Guangdong-Hong Kong-Macao Greater Bay Area cities and their surroundings. Applied Energy, 2018, 228: 1683-1692.

[39] 刘伟玲, 张林波, 龚斌, 王丽霞, 张继平, 张丽丽. 深圳市土地利用碳排放环境库兹涅茨曲线协整分析. 水土保持研究, 2013, 20 (4) : $172-178$.

[40] Liu L, Wu T, Li S S, de Jong M, Sun Y H. The drivers of local environmental policy in China: an analysis of Shenzhen's environmental performance management system, 2007 - 2015. Journal of Cleaner Production, 2017, 165: 656-666.

[41] Sun P W, Zhang N, Zuo J, Mao R C, Gao X F, Duan H B. Characterizing the generation and flows of building interior decoration and renovation waste: a case study in Shenzhen City. Journal of Cleaner Production, 2020, 260: 121077.

[42] 张力小, 张鹏鹏, 郝岩, 唐守娟, 刘耕源. 城市食物-能源-水关联关系: 概念框架与研究展望. 生态学报, 2019, 39(4) : 1144- 1153.

[43] 陈龙, 孙芳芳, 黄涛. 深圳市生态资源消耗与经济增长的脱钩关系研究. 生态经济, 2019, 35(3): 152-157.

[44] Walker R V, Beck M B, Hall J W, Dawson R J, Heidrich O. The energy-water-food nexus: strategic analysis of technologies for transforming the urban metabolism. Journal of Environmental Management, 2014, 141: 104-115.

[45] 李志强, 钱雨果, 周伟奇, 王静. 从“增量扩张” 到“存量优化”: 深圳市景观格局内部动态与优化. 生态学报, 2021 , 41 (5): 1738-1746.

[46] Merino-Saum A, Baldi M G, Gunderson I, Oberle B. Articulating natural resources and sustainable development goals through green economy indicators: a systematic analysis. Resources, Conservation and Recycling, 2018, 139: 90-103. 